\title{
EFFECT OF TRAFFIC POSITION ACCURACY FOR CONDUCTING SAFE AIRPORT SURFACE OPERATIONS
}

\author{
Denise R. Jones, Lawrence J. Prinzel, III, Randall E. Bailey, and Jarvis J. Arthur, III \\ National Aeronautics and Space Administration, Hampton, VA \\ James R. Barnes, Booz Allen Hamilton Engineering Services, LLC, Hampton, VA
}

\begin{abstract}
The Next Generation Air Transportation System (NextGen) concept proposes many revolutionary operational concepts and technologies, such as display of traffic information and movements, airport moving maps (AMM), and proactive alerts of runway incursions and surface traffic conflicts, to deliver an overall increase in system capacity and safety. A piloted simulation study was conducted at the National Aeronautics and Space Administration (NASA) Langley Research Center to evaluate the ability to conduct safe and efficient airport surface operations while utilizing an AMM displaying traffic of various position accuracies as well as the effect of traffic position accuracy on airport conflict detection and resolution (CD\&R) capability. Nominal scenarios and off-nominal conflict scenarios were conducted using 12 airline crews operating in a simulated Memphis International Airport terminal environment. The data suggest that all traffic should be shown on the airport moving map, whether qualified or unqualified, and conflict detection and resolution technologies provide significant safety benefits. Despite the presence of traffic information on the map, collisions or near collisions still occurred; when indications or alerts were generated in these same scenarios, the incidences were averted.
\end{abstract}

\section{Introduction}

The Next Generation Air Transportation System (NextGen) vision transforms the air transportation system to meet the projected growth in aircraft operations expected in the 2025 time-frame in a safe, efficient, and reliable manner [1]. NextGen is envisioned to remove many of the constraints in the current air transportation system, support a wider range of operations, and deliver an overall increase in system capacity and safety. Emerging NextGen operational concepts [2], such as trajectory-based airborne and surface operations, equivalent visual operations, and high-density arrival and departure operations, represent a revolutionary approach to air traffic management; as a result, a dramatic shift in the tasks, roles, and responsibilities for the flight deck are required to ensure a safe, sustainable air transportation system. A net-centric infrastructure will enable a safe and efficient airport surface environment by providing the information necessary to enable improved flight deck and ground vehicle situational awareness through display of traffic information and movements, airport moving maps (AMM), and proactive alerts of runway incursions and surface traffic conflicts.

The National Aeronautics and Space Administration (NASA) is committed to developing technologies, data, and guidelines that may eliminate safety barriers in the terminal maneuvering area that would otherwise constrain the NextGen transformation. A Conflict Detection and Resolution (CD\&R) concept has been developed that leverages advances in flight deck displays and technologies to promote enhanced surface and airborne traffic awareness with associated flight deck alerting concepts for safety assurance. These concepts employ continual ownship and traffic data monitoring and algorithms to detect potential conflicts on the runway and during taxi operations for aircraft and surface vehicles [3-10].

A high-fidelity full-motion piloted simulation study was conducted to evaluate the ability to conduct safe and efficient airport surface operations while utilizing an AMM displaying traffic of various position accuracies as well as the effect of traffic position accuracy on CD\&R capability. The evaluation included approach, departure, and runway incursion scenarios, two AMM conditions, and traffic transmitting various levels of horizontal position accuracy. This paper describes the test set-up, method, and quantitative and qualitative results. 


\section{System Description}

\section{Simulator}

This research was conducted in the Research Flight Deck (RFD) simulator at NASA Langley Research Center (LaRC) (Figure 1) which is a highfidelity, 6 degrees-of-freedom motion-based large commercial aircraft simulator with full-mission capability and advanced glass flight deck displays. Operations were conducted at the Memphis International (KMEM) airport. The out-the-window (OTW) scene included realistic taxiways and runways with appropriate markings, airport lighting, and other aircraft in simulated visibility conditions and provided approximately $20 / 40$ visual acuity with a collimated $200^{\circ}$ horizontal by $40^{\circ}$ vertical field of view at 26 pixels per degree resolution. All standard audio call-outs were issued.

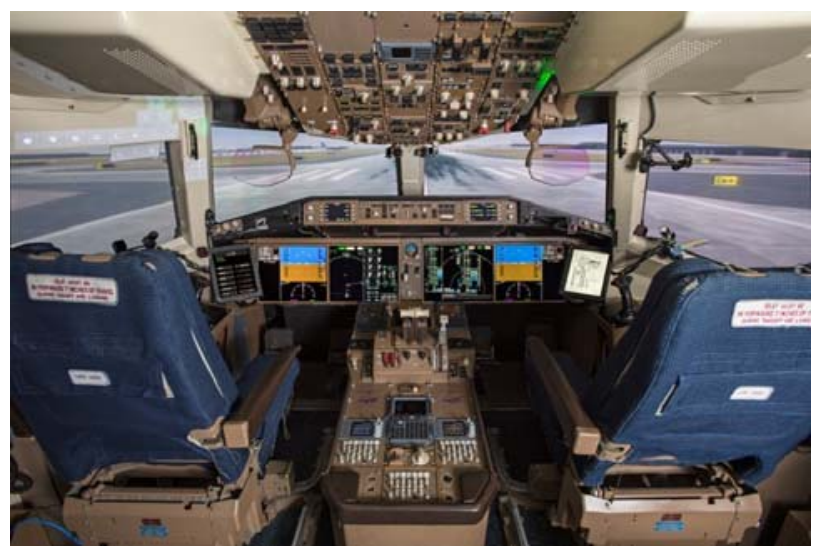

Figure 1. RFD Flight Deck

The RFD is equipped with dual commercial head-up displays; however, they were not utilized for this research.

As shown in Figure 1, the simulator had four large main instrument panel displays referred to as: (left to right) pilot's Primary Flight Display (PFD), pilot's Navigation Display (ND), co-pilot's ND, and co-pilot's PFD. The four display panels were liquid crystal displays with 13.25 inch $x 10.5$ inch viewable area at $1280 \times 1024$ resolution.

Two electronic flight bags (EFBs) were installed. Each provided a display resolution of 1024 x 768 pixels over a 10.4 inch diagonal area. The EFBs were mounted outboard of the PFDs and were used as the pilot's interface for AMMs and charts. The EFBs were located within the pilot's primary field-of-view as per the Federal Aviation Administration (FAA) Advisory Circular (AC) 25$11 \mathrm{~A}[11]$.

\section{Flight Deck Displays}

The PFD and ND were modeled after current state-of-the-art production aircraft. The ND was split, showing a half-screen navigational display and halfscreen Engine Indication and Caution Alerting System.

\section{Electronic Flight Bag Display}

The EFB was used for the following functions: charts, checklists, and AMM. Test subjects interacted with the EFB through either the bezel buttons or a touch screen interface.

The charts function provided the ability to display arrival, approach, departure, and airport charts. Pan/zoom (full page, half of the diagram, one fourth of the diagram) capability was provided.

The checklists function provided the capability to display all aircraft checklists.

The AMM was only displayed on the EFB. Range control for the AMM was provisioned at 0.4 nautical miles $(\mathrm{nm}), 0.8 \mathrm{~nm}, 1.6 \mathrm{~nm}$, and $3.2 \mathrm{~nm}$. The basic AMM display included an airport layout showing runways, taxiways, and buildings. Surface ( $\tan )$ and airborne (cyan) traffic icons were shown along with ownship position (white chevron). The AMM was generated using a KMEM airport geographic database developed to RTCA standards [12]. In this document, all figures of the AMM are shown without the surrounding EFB bezel button structure. Specific AMM configurations used for the study are provided in the Test Method section below.

\section{Air Traffic Control Simulation}

Air traffic control (ATC) instructions and pilot requests and replies were simulated to increase the fidelity of the terminal area environment simulation and provide normal pilot workload demands. All ATC and other aircraft radio messages were issued via a speech generation system. The messages were played through the intercommunication system or 
through the flight deck speaker system when the ownship and simulated traffic reached specified locations or timings to coincide with the scenario task. The subject pilots were asked to provide radio replies, when requested by the ATC messages, as per normal operating procedures. Human back-up was present in the event that additional or unscripted communication or clarification was necessary.

\section{Conflict Detection and Resolution}

A CD\&R system was active during the testing. This system was developed based on Safety, Performance, and Interoperability Requirements (SPR) for Enhanced Traffic Situational Awareness on the Airport Surface with Indications and Alerts (SURF IA) specifications [13] as well as NASA CD\&R research. SURF IA specifications were used to develop the runway conflict detection portion of the CD\&R system.

\section{SURF IA}

SURF IA identifies potential runway conflicts that involve aircraft or vehicles in the airport maneuvering area, within $3 \mathrm{~nm}$ of the runway threshold and 1,000 $\mathrm{ft}$ above field elevation (AFE) and generates both indications and alerts for display to the flight crew. SURF IA utilizes traffic surveillance information obtained from Automatic Dependent Surveillance - Broadcast (ADS-B)-In and generates indications and alerts based on the aircraft/vehicle states during same runway, very closely spaced parallel runway, and intersecting runway operating configurations. Six types of aircraft operational states are defined: 1) taxiing on a taxiway toward a hold line or stopped at a hold line; 2) entering or crossing a runway (not lined up with runway); 3) takeoff; 4) approach; 5) after landing roll-out on runway (e.g., less than or equal to $40 \mathrm{kts}$ ); and, 6) stopped or taxiing along a runway. To prevent inappropriate crew responses during departure, indications and alerts are inhibited above 80 kts. The SURF IA application does not currently address taxiway or low altitude air-to-air conflicts, directive alerting, and is not intended for use on helicopters or vehicles.

\section{NASA CD\&R Research}

NASA CD\&R research has included development of algorithms to identify potential traffic conflicts at low altitudes near the airport, on the runway, and during taxi operations for multiple classes of aircraft and surface vehicles [14]. Since SURF IA does not currently include specifications for taxi conflicts, NASA research specific to taxi conflict detection was used to develop the taxi conflict detection portion of the CD\&R system used for this testing.

The NASA taxi conflict monitor was designed to detect and alert for ground taxi conflicts in the airport movement area by computing distances between the ownship and traffic, closing speeds, time to closest point of approach and other parameters to determine if criteria and thresholds are met for issuing alerts. Indications are not currently generated for taxi conflict situations.

\section{Indications and Alerts}

Indications and alerts (IAs) notified the flight crew of potentially hazardous situations and were presented on the AMM. IA specifications are defined in [13].

Indications were intended to generate pilot awareness and situation assessment by highlighting the runway and traffic status as relevant to ownship operations. Indications identified operational conditions that were generally normal, yet relevant for runway safety and could be a precursor to a nonnormal situation. Only visual annunciations were required and used for indications. Indications were only issued for runway conflict situations. Two types of indications were utilized.

A traffic indication (TI) highlighted a potential runway traffic collision/hazard that could emerge in the near future. TIs were intended to increase the flight crews' awareness of the relevant runway traffic. The flight crew could proceed with the intended operation after a brief assessment of the situation and if appropriately cleared. A TI was displayed on the AMM as an enlarged traffic symbol for the relevant traffic surrounded by a dashed circle in the same color as the traffic symbol and an identification tag that showed flight identification and ground speed in knots. A status message ("Traffic") was displayed at the bottom of the AMM along with the estimated distance to the traffic in nautical miles until below $0.1 \mathrm{~nm}(600 \mathrm{ft})$, then displayed in feet.

A runway status indication (RSI) identified whether the runway that the ownship was 
approaching or using was in-use or occupied by other traffic and was not suitable for entering, takeoff, or landing. Before proceeding, the crew should ensure they had the appropriate clearance and the indicated traffic was not a factor. An RSI was displayed on the AMM (Figure 2) in the same manner as a TI with the addition of a solid blue line outlining the relevant runway.

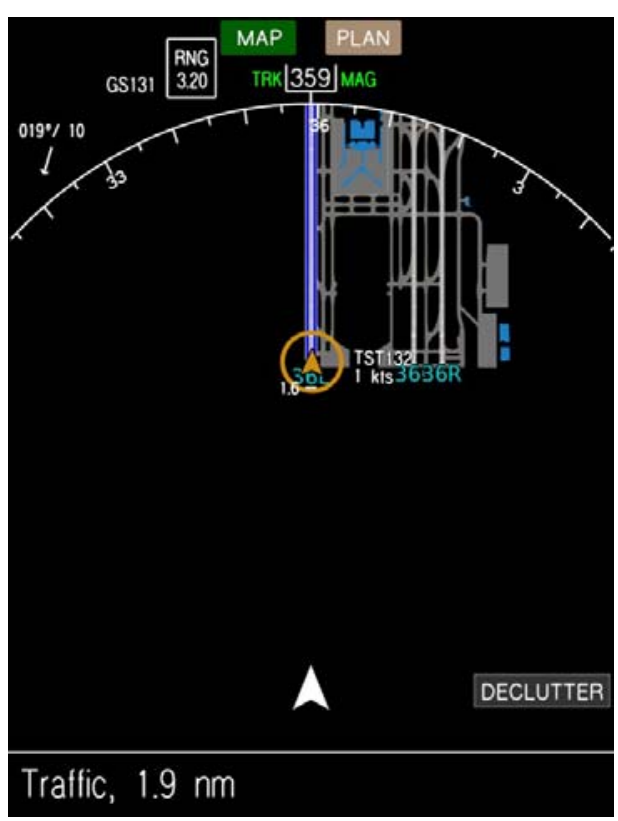

\section{Figure 2. AMM Showing Runway Status Indication}

Alerts identified potential collision hazards which require immediate awareness by the flight crew and may require timely action or response to avoid a collision. Alerts had priority over indications. Auditory and visual annunciations were required for alerts. Alerts were issued for both runway and taxi conflict situations. A two-level alerting scheme was utilized.

Caution alerts were generated for conditions that required immediate flight crew awareness and subsequent flight crew response. Generally, caution alerts were issued with sufficient time to allow a crew to evaluate the situation and to prepare to respond. A caution alert was displayed on the AMM as an enlarged yellow traffic symbol surrounded by a yellow circle for the relevant traffic, an identification tag that showed flight identification and ground speed in knots, and a yellow line around the relevant runway, if applicable. An alert message ("Caution,
Traffic") was displayed at the bottom of the AMM in yellow text along with the estimated distance to the traffic. An audible annunciation was also made ("Caution, Traffic, Caution, Traffic").



Figure 3. AMM Showing Warning Alert

Warning alerts were issued for conditions that required immediate flight crew awareness and immediate flight crew response. Warning alerts could occur without preceding caution alerts. A warning alert was displayed in the same manner on the AMM as a caution alert, except the warning was associated with the color red, a square was used to surround the traffic symbol, and the alert message was "Warning, Traffic" (Figure 3).

\section{Surveillance Data}

The quality and accuracy of reported traffic surveillance data are critical to the integrity of the AMM traffic displays and the CD\&R capability. The traffic position accuracy was simulated as dependent upon the Global Positioning System (GPS) measurement errors. A Gauss-Markov process modeled the time correlation between successive position measurement errors [15]. It was assumed that ADS-B would be used as the means for transmitting (ADS-B Out) and receiving (ADS-B In) these GPS-based aircraft surveillance data. Although ADS-B transmission qualities and effects were not modeled for this study, the traffic positional data was 
updated at one hertz to simulate ADS-B transmission rates. Latency effects, transmission line-of-sight, bandwidth blockage, and vertical position accuracy were not modeled.

The Navigation Accuracy Category for Position (NACp) describes the accuracy of positional information. NACp values range from 0 to 11 [16]. The NACp categories of 8 and higher are listed in Table 1 with their associated horizontal Estimated Position Uncertainty (EPU) values.

Table 1. NACp Horizontal Accuracy Bound

\begin{tabular}{|c|c|}
\hline NACp & $\begin{array}{l}\text { 95\% Horizontal Accuracy - Estimated } \\
\text { Position Uncertainty (EPU) }\end{array}$ \\
\hline 8 & EPU $<92.6 \mathrm{~m}(0.05 \mathrm{~nm}, 305.6 \mathrm{ft})$ \\
\hline 9 & EPU $<30 \mathrm{~m}(99 \mathrm{ft})$ \\
\hline 10 & EPU $<10 \mathrm{~m}(33 \mathrm{ft})$ \\
\hline 11 & EPU $<3 \mathrm{~m}(9.9 \mathrm{ft})$ \\
\hline
\end{tabular}

The FAA has issued an ADS-B Out Final Rule [17] which includes performance standards. The rule states that EPU of the reported position must be less than $0.05 \mathrm{~nm}$, which is equivalent to NACp 8 .

Both DO-289 (Minimum Aviation System Performance Standards for Aircraft Surveillance Applications) [18] and DO-322 (SPR for Airborne Traffic Situational Awareness for Surface Operations (ATSA-SURF) Application) [19] define the minimum requirement for horizontal position accuracy for depiction of ground traffic on an AMM to be at least $30 \mathrm{~m}$ within $95 \%$ containment bounds, equivalent to NACp 9. Requirements for other navigation parameters are also specified in these documents but will not be addressed in this paper. The positional surveillance accuracy of NACp 8 , as specified in the ADS-B Out rule, is not sufficient to support the depiction of traffic information on an AMM display, per DO-322, although possible mitigation strategies have been proposed (e.g., RTCA DO-322, Appendix D) [19].

The SURF IA SPR has proposed horizontal position accuracy requirements [13] for the SURF IA function. Through analysis, the SPR identified that to meet safety requirements, horizontal position accuracy when on the airport surface needs to be at least $10 \mathrm{~m}$ within $95 \%$ containment bounds (NACp 10) to allow indications and alerts to be issued for traffic at virtually all airports in the National Airspace System. Validation of these requirements is on-going.

To span the ADS-B Out Final Rule, ATSASURF, and SURF IA position accuracy reporting requirements (Table 2), traffic position accuracies equivalent to NACp 8, 9, 10, and 11 levels were used for this test. Truth data, with no accuracy errors, was also recorded. The capability was provided to enable selection of the desired accuracy level (e.g. NACp 8, NACp 9, etc.) for simulated traffic individually. For this study, traffic transmitting horizontal position accuracy equivalent to NACp 8 definitions was considered unqualified traffic. Traffic transmitting position accuracy equivalent to NACp 9 and higher was considered qualified traffic.

Table 2. NACp Accuracy Requirements

\begin{tabular}{|c|c|c|c|}
\hline NACp & $\begin{array}{c}\text { ADS-B Out } \\
\text { Final Rule }\end{array}$ & $\begin{array}{c}\text { Display Per } \\
\text { DO-322 } \\
\text { (ATSA-SURF) }\end{array}$ & $\begin{array}{c}\text { IAs Per } \\
\text { DO-323 } \\
\text { (SURF IA) }\end{array}$ \\
\hline 8 & Yes & No & No \\
\hline 9 & Yes & Yes & No \\
\hline 10 & Yes & Yes & Yes \\
\hline 11 & Yes & Yes & Yes \\
\hline
\end{tabular}

\section{Test Method}

The objectives of the testing were to evaluate the ability of a subject crew to conduct safe and efficient airport surface operations while utilizing an AMM displaying traffic of various position accuracies as well as the effect of traffic position accuracy on CD\&R capability. The evaluation included approach, departure, and runway incursion scenarios and two AMM conditions with traffic transmitting various levels of horizontal position accuracy.

\section{Evaluation Pilots}

Twenty-four commercial pilots served as participants for the research. The test subjects were paired by airline and role (Captain, First Officer) to ensure crew coordination and cohesion with regard to terminal and surface standard operational procedures. All pilots held an Airline Transport Pilot rating. The Captains had an average of over 17,000 flight hours with 25 years of commercial experience. The First Officers had an average of over 13,000 flight hours with 20 years of commercial experience. 


\section{AMM Display Conditions}

These AMM conditions were chosen to evaluate the effects of displaying all airport traffic on an AMM (Map B) versus displaying only qualified traffic on an AMM (Map A).

The Map A condition consisted of the basic AMM format displaying qualified traffic only (traffic reporting a horizontal position accuracy of NACp 9 and higher). As a result, the locations of some of the airport traffic (those reporting horizontal position accuracy of NACp 8) were not displayed. The only method of acquiring the NACp 8 reporting traffic was visually, OTW.

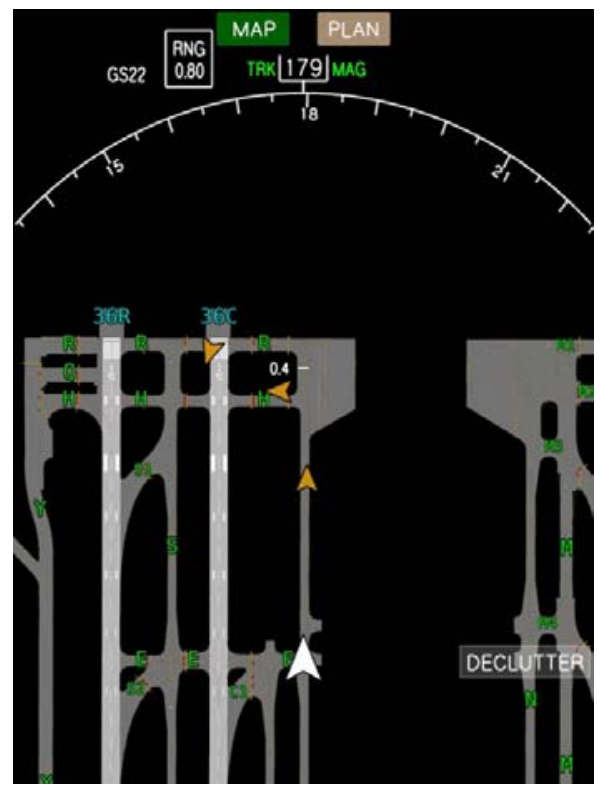

\section{Figure 4. AMM Display Showing All Traffic}

The Map B condition consisted of the basic AMM format displaying both qualified and unqualified traffic (traffic reporting a horizontal position accuracy of NACp 8) (Figure 4); therefore, all airport traffic was displayed on the AMM. As position accuracy decreased, the location of traffic symbols could vary from the traffic's actual location. For example, the traffic symbol for an aircraft transmitting a position accuracy of NACp 8 could be displayed $305 \mathrm{ft}$ or more from the aircraft's actual location, giving a potentially misleading indication of the traffic's location.

The NACp levels of the traffic position reports were not indicated to the crew by icon shape or color on the AMM concepts

\section{Test Matrix}

A total of 10 trials (8 nominal, 2 off-nominal) were conducted by each crew. All trials were conducted in daytime $1,800 \mathrm{ft}$ visibility.

Nominal trials were conducted to evaluate the effect of displaying traffic transmitting various position accuracies on the AMM during airport surface operations. Traffic was transmitting position accuracy ranges from NACp 8 to NACp 11. Two AMM conditions (Map A and Map B) were evaluated across subjects during two approach and two departure scenarios, for a total of eight nominal trials.

For the approach scenarios, the ownship was initialized approximately $5 \mathrm{~nm}$ from the runway threshold for a standard auto-land approach. After landing and roll-out, the aircraft was to exit the runway and taxi to the ramp via the designated taxi route. For the departure scenarios, the subject crew began taxi from the ramp, taxied to the departure runway via the designated taxi route, and executed a takeoff after receiving the appropriate clearance. The trial terminated once the aircraft reached approximately $1000 \mathrm{ft}$ AFE. The pilots were requested to maintain a standard taxi speed of $15 \mathrm{kts}$ whenever feasible.

Off-nominal trials were conducted between subjects to evaluate the potential safety implications of traffic position accuracy on airport surface CD\&R capability. Each crew completed two off-nominal trials as Test Runs 6 and 10 of 10 trials.

For six of the crews, the objective was to evaluate the impact of displaying qualified versus unqualified traffic on the AMM during conflict situations. These crews were given a taxi crossing and departure conflict scenario (see below for description), using either Map A or B display condition. The conflict traffic was transmitting NACp 8 position accuracy for all these trials; therefore, IAs were not issued. When using Map A, the conflict traffic was not displayed on the AMM and only could be viewed OTW. When using Map B, the conflict traffic was displayed on the AMM, albeit with NACp of 8 accuracy, and could also be viewed OTW.

For the other six crews, the objective of the offnominal trials was to evaluate the impact of receiving 
versus not receiving IAs for traffic displayed on the AMM during conflict situations. These crews were given a taxi crossing and departure conflict scenario using the Map A display condition. The conflict traffic was transmitting either NACp 9 or NACp 10 position accuracy and, therefore, was always displayed on the AMM. IAs were not issued for traffic transmitting NACp 9 position accuracy, but were issued for traffic transmitting NACp 10 position accuracy.

\section{Conflict Scenarios}

Two conflict scenarios were utilized. Every effort was made to produce similar timings; however, a certain amount of variability was naturally introduced due to the maneuvering conducted by the pilot (e.g., taxi speed).

\section{Taxi Crossing Conflict Scenario}

An approach and departure flow was simulated. Traffic was approaching Runway 36C (Figure 5, green route) and Runway 36L (Figure 5, orange route), spaced $5 \mathrm{~nm}$ apart and staggered between runways. This traffic landed, exited the runway, and taxied to the terminal via the routes shown in Figure 5. There was a departure flow using Runway $36 \mathrm{R}$ via the blue colored route shown in Figure 5. Other static traffic was placed in strategic locations to add interest to the scenario.

At the beginning of this scenario, the ownship was parked on the ramp near Taxiway M6 facing Taxiway N. The flight crew was cleared to taxi to Runway 36C via Taxiways N, P, S, and R (Figure 5, magenta route), holding short of Runway $36 \mathrm{C}$. As the ownship approached the Runway 36C hold line, an aircraft was landing on the runway. The subject crew was cleared to cross Runway 36C when the landing traffic was approximately $8,000 \mathrm{ft}$ away.

This scenario tested the incursion situation where an aircraft taxis across a runway even though another aircraft is landing on the same runway. In this case, the subject crew was mistakenly cleared to cross the runway, resulting in a potential collision unless action was taken.

\section{Departure Conflict Scenario}

A southern approach and departure flow was simulated. Traffic was approaching Runway 18R

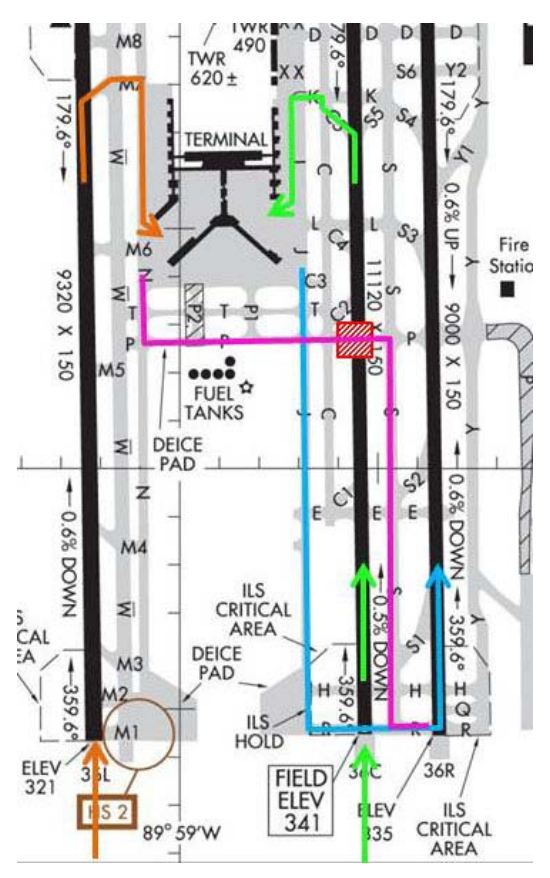

Figure 5. Taxi Crossing Conflict Scenario

(Figure 6, orange route) and Runway 18L (Figure 6, blue route), spaced $5 \mathrm{~nm}$ apart and staggered between runways. This traffic landed, exited the runway, and taxied to the terminal via the routes shown in Figure 6 . There was a departure flow using Runway $18 \mathrm{C}$ via the magenta colored route shown in Figure 6.

One aircraft taxied from the ramp via the green route shown in Figure 6 and held short of Runway $18 \mathrm{C}$ at Taxiway D. This aircraft was intended to be a distraction for the subject crew. Other static traffic was placed in strategic locations to add interest to the scenario.

At the beginning of this scenario, the ownship was parked on the ramp facing Taxiway J. The flight crew was cleared to taxi to Runway $18 \mathrm{C}$ via Taxiways J, K, C, and C8 (Figure 6, magenta route), holding short of Runway 18C. As the ownship was cleared and was taxied onto the runway, another aircraft taxied from the ramp (Figure 6, yellow route) and held short of Runway $18 \mathrm{C}$ at Taxiway L. As the ownship began its departure roll, the aircraft holding on Taxiway $\mathrm{L}$ crossed the runway in front of the ownship. Taxiway L is $4,500 \mathrm{ft}$ from the Runway 18C threshold.

This scenario tested the incursion situation where an aircraft taxis across a runway in front of a departing aircraft. The traffic for this scenario, 
instead of holding short, taxied across Runway 18C without clearance as the ownship began its departure.

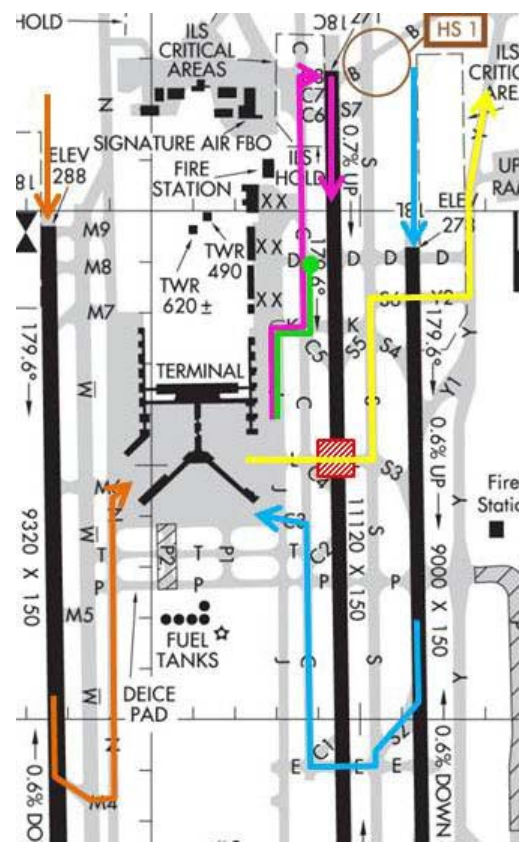

Figure 6. Departure Conflict Scenario

\section{Procedure}

Prior to the testing phase, each test subject participated in a briefing and training session. The training included general simulator characteristics and specific items and procedures related to the testing, such as Map Conditions A and B, traffic position accuracy, and CD\&R capability.

During the training for the CD\&R capability, the flight crews were trained to abort if a warning alert was generated during departure, go-around if a warning alert was generated on approach, and stop if a warning alert was generated during taxi. They were not required to take evasive action when an indication or caution alert was issued.

Before each trial, the flight crew was briefed on the run conditions, e.g., approach or departure, visibility, winds, display condition in use, and ATC radio frequencies.

The test runs were documented via audio, video, and digital data recordings, and post-run and post-test questionnaires.

\section{Test Metrics}

For conflicts, a near collision was counted if the center-of-gravity (CG) of the two aircraft were $<300$ $\mathrm{ft}$ apart laterally and vertical separation was $<200 \mathrm{ft}$. A collision was counted if the aircraft CGs were < $150 \mathrm{ft}$ apart laterally and vertical separation was $<$ $100 \mathrm{ft}$.

\section{Results}

A summary of quantitative and qualitative results is presented. All data are referenced from the aircraft CG. All statistically significant results are presented at the $\mathrm{p}<0.05$ level. Nominal scenario results are included in the qualitative results section.

\section{Off-Nominal Scenario Results}

As described in the Test Method section, the test objective for six of the crews was to evaluate the impact of displaying qualified versus unqualified traffic on the AMM during conflict situations. These crews were given a taxi crossing and departure conflict scenario, using either Map A or B display condition. The conflict traffic was transmitting NACp 8 position accuracy for all of these trials; therefore, IAs were not issued. When using Map A, the conflict traffic was not displayed on the AMM and could only be viewed OTW, if possible for the visibility conditions. When using Map B, the conflict traffic was displayed on the AMM and could also be viewed OTW.

When using the Map A condition, action was only taken to avoid the conflict traffic on one of six trials (17\%) (Table 3). The flight crew was not aware of the conflict traffic on five of these trials and continued the operations, resulting in four collisions and one near collision. On one trial, after seeing the traffic OTW on takeoff roll, a high speed rejected takeoff (131 kts) was conducted. The aircraft stopped just before reaching the traffic, resulting in a near collision.

When using the Map B condition, action was taken on four of six trials (67\%) (Table 3). For the taxi crossing scenario, one crew crossed the runway, unaware of the conflict traffic, resulting in a collision. This crew viewed the AMM for traffic, zooming out to the largest map scale; the map was 
scanned just before the traffic came into view. For the departure scenario, the crew that departed saw the traffic on the AMM but was not sure if the traffic was on the runway due to the traffic's position accuracy (aircraft symbol was "dancing" on the AMM).

Table 3. Off-Nominal Scenario Results, Map Condition Focus

\begin{tabular}{|c|c|c|}
\hline & Taxi Crossing & Departure \\
\hline $\begin{array}{l}\text { Map A, } \\
\text { NACp } 8\end{array}$ & $\begin{array}{l}3 \text { crossed: } \\
2 \text { collisions, } \\
1 \text { near collision }\end{array}$ & $\begin{array}{l}2 \text { departures, } \\
1 \text { high speed reject: } \\
2 \text { collisions, } \\
1 \text { near collision }\end{array}$ \\
\hline $\begin{array}{l}\text { Мap B, } \\
\text { NACp } 8\end{array}$ & $\begin{array}{l}1 \text { crossed, } \\
2 \text { held short: } \\
1 \text { collision }\end{array}$ & $\begin{array}{l}1 \text { departure, } \\
2 \text { held in position: } \\
1 \text { collision }\end{array}$ \\
\hline
\end{tabular}

For the other six crews, the objective of the offnominal trials was to evaluate the impact of receiving IAs versus not receiving them for traffic displayed on the AMM during conflict situations. These crews were given a taxi crossing and departure conflict scenario using the Map A display condition. The conflict traffic was transmitting either NACp 9 (no IAs) or NACp 10 (IAs issued) position accuracy and, therefore, was always displayed on the AMM.

When the conflict traffic was transmitting NACp 9 accuracy, no action was taken on three of six trials (50\%) (Table 4). Two of the taxi crossing trials resulted in collisions; the crews did not see the traffic on the AMM and crossed the runway. Two of the departure trials also resulted in collisions. During one of the departure trials, the crew saw the conflict traffic on the AMM but were not sure if the traffic was on the runway due to the traffic's position accuracy so they continued the departure. During the other departure trial, the crew saw the traffic OTW and conducted a high speed rejected takeoff (132 $\mathrm{kts})$, having to veer to the right of the conflict traffic, which resulted in a collision.

When the conflict traffic was transmitting NACp 10 accuracy, action was taken to avoid the conflict on all six trials (Table 4). For the three taxi crossing trials, action was based on receiving RSIs. For the three departure trials, action was based on receiving a warning, an RSI, and viewing the traffic on the AMM.
Table 4. Off-Nominal Scenario Results, NACp Focus

\begin{tabular}{|l|l|l|}
\cline { 2 - 3 } \multicolumn{1}{c|}{} & \multicolumn{1}{c|}{ Taxi Crossing } & \multicolumn{1}{c|}{ Departure } \\
\hline $\begin{array}{l}\text { Map A, } \\
\text { NACp 9 }\end{array}$ & $\begin{array}{l}2 \text { crossed, } \\
1 \text { held short: } \\
2 \text { collisions }\end{array}$ & $\begin{array}{l}\text { 1 departure, 2 reject: } \\
2 \text { collisions }\end{array}$ \\
\hline $\begin{array}{l}\text { Map A, } \\
\text { NACp 10 }\end{array}$ & 3 held short & $\begin{array}{l}\text { 1 held in position, } \\
2 \text { rejected }\end{array}$ \\
\hline
\end{tabular}

\section{Taxi Crossing Off-Nominal Details}

The details of the taxi crossing offnominal/conflict trials are presented in Table 5.

The data shown that, with all traffic displayed and with an increase in position accuracy, the number of collisions/near collisions were reduced. All trials in which the conflict traffic was not displayed on the AMM (Map A condition with conflict traffic transmitting NACP 8 position accuracy) resulted in a collision or near collision. Collisions/near collisions were reduced when all the traffic was displayed on the AMM (Map B condition and Map A condition with conflict traffic transmitting NACp 9 accuracy). When IAs were issued (conflict traffic transmitting NACp 10 accuracy), there were no collisions or near collisions.

In Table 5, all distances are referenced to the $\mathrm{CG}$ (the CG of the ownship was $72.8 \mathrm{ft}$ from the nose of the aircraft). For all trials but one in which the aircraft held short of the hold line (HL), the nose was well behind the hold line (40 to $98 \mathrm{ft}$ ). In this one case, the nose did cross the hold line (technically a runway incursion) but stopped $\sim 150 \mathrm{ft}$ before reaching the runway edge.

IAs were possible on the conflict traffic on three taxi crossing trials (transmitting NACp 10 accuracy). A TI was generated on one trial, $\sim 3.4 \mathrm{~nm}$ from the traffic, $212 \mathrm{ft}$ prior to crossing the hold line. An RSI was generated on all three trials, $10,341 \mathrm{ft}$ mean distance from the traffic (standard deviation $(\sigma)=$ $7,373 \mathrm{ft}), 206 \mathrm{ft}$ mean distance $(\sigma=13.5 \mathrm{ft}$ ) prior to crossing the hold line. The large standard deviation was caused by the wide variability in taxi operations. One crew taxied slower than the planned $15 \mathrm{kts}$ and reached the runway hold line when the traffic was only 2,586 ft from the ownship. In contrast, another crew generally taxied faster than the planned $15 \mathrm{kts}$ 
and reached the runway hold line before conditions were met for IAs (TI and RSI later issued).

IAs were also generated for other traffic during the off-nominal trials. TIs were generated on all 12 taxi crossing trials for an aircraft that was landing behind the conflict traffic as the ownship was crossing the runway (distance from traffic, mean $(\mu)$ $=35,222 \mathrm{ft}, \sigma=74 \mathrm{ft}$ ). One crew received an RSI on this same aircraft (distance from traffic, 17,147 ft). Three crews also received IAs on an aircraft parked at a gate in the ramp area (one caution, $49 \mathrm{ft}$ from traffic; two warnings, $\mu=265 \mathrm{ft}, \sigma=1.7 \mathrm{ft}$ from traffic). Even though the static traffic was transmitting NACp 10 position accuracy, for these trials, there was a larger error on the position data as calculated by the surveillance data model, placing the aircraft closer to the path of the ownship. Since aircraft can be closer together in the congested ramp area, perhaps IAs should be inhibited in the ramp area to minimize nuisance alerting caused by position data inaccuracies.

Table 5. Taxi Crossing Scenario Results

\begin{tabular}{|c|c|c|c|c|c|c|}
\hline Map & NACp & Action & Outcome & $\begin{array}{c}\text { CPA* } \\
\text { (feet) }\end{array}$ & CG Dist to HL (feet) & Traffic Awareness \\
\hline A & 8 & Crossed Runway & Collision & 21.3 & N/A & Not Aware \\
\hline A & 8 & Crossed Runway & Near Collision & 262.9 & N/A & Not Aware \\
\hline A & 8 & Crossed Runway & Collision & 104.1 & N/A & Not Aware \\
\hline B & 8 & Held Short & Stopped & 385.3 & 134.5 & AMM \\
\hline B & 8 & Held Short & Stopped & 452.9 & 114.6 & AMM \\
\hline B & 8 & Crossed Runway & Collision & 154.4 & N/A & $\begin{array}{c}\text { Not Aware - early } \\
\text { map check }\end{array}$ \\
\hline A & 9 & Held Short & Stopped & 400.1 & 156.5 & AMM \\
\hline A & 9 & Crossed Runway & Collision & 161.9 & N/A & Not Aware \\
\hline A & 9 & Crossed Runway & Collision & 63.9 & N/A & Not Aware \\
\hline A & 10 & Crossed HL & Stopped & 299.1 & 19.0 & RSI \\
\hline A & 10 & Held Short & Stopped & 398.2 & 113.6 & RSI \\
\hline A & 10 & Held Short & Stopped & 471.4 & 171.1 & RSI \\
\hline
\end{tabular}

* CPA - Closest Point of Approach

Table 6. Departure Scenario Results

\begin{tabular}{|c|c|c|c|c|c|c|}
\hline Map & $\mathrm{NACp}$ & Action & Outcome & CPA (feet) & RTO GS* & Traffic Awareness \\
\hline A & 8 & Took-off & Collision & 44.0 & N/A & Not Aware \\
\hline A & 8 & TO roll & $\begin{array}{l}\text { RTO - Near } \\
\text { Collision }\end{array}$ & 209.9 & 131 & OTW \\
\hline $\mathrm{A}$ & 8 & Took-off & Collision & 151.3 & N/A & Not Aware \\
\hline B & 8 & Held Position & Stopped & $4,054.4$ & N/A & AMM \\
\hline $\mathrm{B}$ & 8 & Held Position & Stopped & $4,214.1$ & N/A & AMM \\
\hline B & 8 & Took-off & Collision & 74.6 & $\mathrm{~N} / \mathrm{A}$ & $\begin{array}{l}\text { Map but unsure due to } \\
\text { accuracy issue }\end{array}$ \\
\hline A & 9 & Start TO & RTO & $2,626.5$ & 35 & AMM \\
\hline A & 9 & TO roll & RTO-Collision & 169.4 & 132 & OTW \\
\hline A & 9 & Took-off & Collision & 128.3 & $\mathrm{~N} / \mathrm{A}$ & $\begin{array}{l}\text { Map but unsure due to } \\
\text { accuracy issue }\end{array}$ \\
\hline A & 10 & Initial roll & Stopped & $4,240.8$ & N/A & AMM \\
\hline A & 10 & Start TO & RTO-Stopped & $1,386.8$ & 93 & Warning \\
\hline A & 10 & Start TO & RTO-Stopped & $3,390.9$ & 47 & RSI, but got Warning \\
\hline
\end{tabular}

*RTO GS - Roll-out Turn-Off Ground Speed 


\section{Departure Off-Nominal Details}

The details of the departure conflict trials are presented in Table 6 .

As with the taxi crossing trials, the number of collisions/near collisions were reduced when all traffic was displayed and position accuracy was increased. All trials in which the conflict traffic was not displayed on the AMM (Map A condition with conflict traffic transmitting NACP 8 position accuracy) resulted in a collision or near collision. Collisions/near collisions were reduced when all the traffic was displayed on the AMM (Map B condition and Map A condition with conflict traffic transmitting NACp 9 accuracy). However, when IAs were issued (traffic transmitting NACp 10 accuracy), there were no collisions or near collisions. The CPA distance varied greatly depending on the action of the pilot. Also, in all trials in which the aircraft took-off, the collision occurred just after liftoff when the aircraft was $40 \mathrm{ft}$ or less above ground level (AGL).

Based on these departure test trials, the pilots rejected the takeoff sooner when traffic awareness occurred based on either viewing the conflict traffic on the AMM or when IAs were issued. By the time the conflict traffic could be viewed OTW, it was too late to conduct a successful high speed rejected takeoff (RTO) resulting in a collision and near collision.

IAs were possible on the conflict traffic on three departure trials (transmitting NACp 10 accuracy). An RSI was generated on one trial prior to receiving a warning alert, 4,268 $\mathrm{ft}$ from the traffic, when traveling $21 \mathrm{kts}$. A warning alert was generated on two trials, $3,898 \mathrm{ft}$ mean distance from the traffic $(\sigma=$ $298 \mathrm{ft})$, when traveling $51 \mathrm{kts}$ mean $(\sigma=15 \mathrm{kts})$. After initiating an RTO/stop, an RSI was generated on all three departure trials (distance from traffic, $\mu=$ $3,338 \mathrm{ft}, \sigma=1,043 \mathrm{ft}$; ground speed, $\mu=25 \mathrm{kts}, \sigma=$ $16 \mathrm{kts})$.

\section{Qualitative Results}

Post-run and post-test questionnaires were administered. Most of the questions were rated on a scale of 1 ("strongly disagree", "low", "not useful") to 7 ("strongly agree", "high", "very useful").
Traffic Awareness and Symbology - Based on post-run responses, the map condition showing all traffic (Map B: $\mu=5.4, \sigma=2.1$ ) was significantly better for traffic awareness than the map condition only showing qualified traffic (Map A: $\mu=3.0, \sigma=$ 2.1). Furthermore, the pilots indicated the presentation of only qualified traffic on the AMM was a greater safety issue $(\mu=6.3, \sigma=1.1)$ than displaying all traffic $(\mu=2.3, \sigma=1.7)$. They also felt the display of only qualified traffic on the AMM will increase the potential for accidents $(\mu=5.5, \sigma=1.4)$ than the display of all traffic $(\mu=2.0, \sigma=1.6)$. When asked if all traffic should be displayed on the AMM or only qualified traffic (transmitting NACp 9 and higher position accuracy levels), 23 of 24 pilots (96\%) responded that all traffic should be displayed (confidence level of $\mu=6.4, \sigma=0.6$, where $7=100 \%$ confidence). These results are consistent with the quantitative performance that shows fewer accidents and incidents with all traffic being displayed.

As one would expect, when the displayed traffic was of lower position accuracy (NACp 8 condition: $\mu$ $=3.0, \sigma=2.1$ vs. NACp 10 condition $: \mu=6.1, \sigma=$ $1.1)$, it was rated significantly lower for traffic awareness. The pilots were asked if the NACp surveillance accuracy should be shown on the AMM. The pilot ratings indicated a slight preference for using different symbology for qualified and unqualified traffic $(\mu=5.4, \sigma=2.0)$ instead of using the same symbology $(\mu=4.5, \sigma=1.7)$ to avoid potentially misleading information (not statistically significant).

For this study, the AMM was located on the EFB outboard of the PFD. The pilots moderately agreed that this was an optimal location for viewing the AMM $(\mu=5.3, \sigma=1.4)$. Several pilots commented that the AMM should be located on a forward display, preferably the ND, or in a central location for viewing by both pilots.

Conflict Awareness and CD\&R Symbology The possibility exists that the flight crew may not be aware of potential conflict traffic when using Map A condition (showing only qualified traffic), particularly if visibility is low and traffic cannot be visually acquired OTW. Based on the post-run responses, the map condition showing all traffic (Map B: $\mu=5.3, \sigma=1.9$ ) was significantly better for detection of potential surface conflicts than the map 
condition only showing qualified traffic (NACp 9 and higher) (Map A: $\mu=1.3, \sigma=0.5$ ).

When the conflict traffic was transmitting NACp 8 position accuracy, the traffic was not displayed on the AMM. The conflict traffic was displayed on the AMM when it was transmitting NACp 9 accuracy; however, IAs were not issued. When transmitting NACp 10 accuracy, the conflict traffic was displayed on the AMM and IAs were issued, providing the most information related to potential surface conflicts. Traffic transmitting more accurate position data (NACp 9: $\mu=3.9, \sigma=2.2$ and NACp 10: $\mu=$ 6.7, $\sigma=0.5$ ) was rated higher for detection of potential surface conflicts than traffic transmitting less accurate data (NACp 8: $\mu=1.3, \sigma=0.5$ ).

The pilots rated the CD\&R symbology as effective in providing information on the conflict traffic $(\mu=6.4, \sigma=1.0)$ and providing a clear indication of the relative location of the conflict traffic $(\mu=6.5, \sigma=0.7)$. Both indications and alerts were helpful in determining critical runway safety information (indications: $\mu=6.3, \sigma=1.2$, alerts: $\mu=$ $6.5, \sigma=0.6$ ), provided additional information over AMM traffic (indications: $\mu=6.2, \sigma=0.9$, alerts: $\mu=$ $6.4, \sigma=0.7$ ), and helped in determining the location and movement of traffic that was relevant to the safety of their own aircraft (indications: $\mu=6.3, \sigma=$ 0.8 , alerts: $\mu=6.4, \sigma=0.7$ ).

The pilots felt there should be a distinction (difference) between the symbology representing traffic qualified for the CD\&R indication and alerting function (traffic transmitting NACp 10 or 11) versus traffic not qualified for that function (traffic transmitting NACp 9 or less) $(\mu=5.3, \sigma=1.7)$.

General - The pilots rated their level of perceived safety higher having the CD\&R system onboard their aircraft during similar operations $(\mu=$ $6.2, \sigma=0.6)$ than without having the system onboard $(\mu=4.8, \sigma=0.9)$ (statistically significant). With a rating of 7 being completely safe, the pilots rated the level of safety felt during runway conflict incidents $(\mu=5.7, \sigma=1.0)$ and during taxi conflict incidents $(\mu$ $=5.9, \sigma=0.8$ ) as moderately high. The pilots were asked to provide suggestions for improvements that would increase the safety of the system. Some of the prevalent comments were: more experience with the system; detailed training, including appropriate map scale per operation; reduce number of menu levels (button pushing) on the EFB; display all traffic on the AMM; provide IAs for all traffic; and directive alerting. Also, the question of qualified vs. unqualified traffic and traffic position accuracy added to mental workload and uncertainty. Some of the best features identified were the display of traffic on the AMM and IAs.

\section{Summary}

A piloted simulation study was conducted to evaluate the ability to conduct safe and efficient airport surface operations while utilizing an AMM displaying traffic of various position accuracies as well as the effect of traffic position accuracy on airport CD\&R capability.

With all traffic displayed and with an increase in position accuracy, the number of collisions/near collisions was reduced. A collision or near collision occurred on each trial in which the conflict traffic was not displayed on the AMM (transmitting NACp 8 accuracy). There were no collisions when IAs were generated on the conflict traffic (transmitting NACp $\geq 10$ accuracy), notifying the flight crew of the potential conflict situation.

The test subjects thought that all the traffic should be displayed on the AMM, regardless of the position accuracy level, to provide a higher level of traffic awareness, increased safety, and to reduce the potential for accidents. There are some concerns with displaying traffic transmitting less accurate NACp 8 position accuracy, however. Due to the level of position uncertainty, pilots were sometimes unsure if the traffic was actually on the runway and continued the operation, resulting in collision.

The CD\&R system was very effective in preventing collisions; no collisions occurred when IAs were generated for conflicting traffic. The current design, however, issued nuisance alerts on traffic in the ramp area due to position accuracy, even at the NACp 10 level.

\section{References}

[1] Joint Planning \& Development Office, 2010, Executive Summary, Integrated Work Plan for the Next Generation Air Transportation System, Version FY13, Washington, DC. 
[2] Joint Planning \& Development Office, 2010, Concept of Operations for the Next Generation Air Transportation System, Version 3.2, Washington, DC.

[3] Jones, D. R., C. C. Quach, and S. D. Young, 2001, Runway Incursion Prevention System -

Demonstration and Testing at the Dallas/Fort Worth International Airport, Proceedings of the $20^{\text {th }}$ Digital Avionics Systems Conference, Daytona Beach, FL.

[4] Jones, D. R., 2002, Runway Incursion Prevention System Simulation Evaluation, Proceedings of the AIAA/IEEE $21^{\text {st }}$ Digital Avionics Systems Conference. Irvine, CA.

[5] Jones, D. R., 2005, Runway Incursion Prevention System Testing at the Wallops Flight Facility, Proceedings of the SPIE Defense \& Security Symposium, Enhanced and Synthetic Vision 2005, Vol. 5802, pp. 47-58.

[6] Jones, D. R. and L. J. Prinzel, III, 2006, Runway Incursion Prevention for General Aviation Operations, Proceedings of the 25th Digital Avionics Systems Conference, Portland, OR.

[7] Jones, D. R., L. J. Prinzel, S. D. Otero, and G. D. Barker, 2009, Collision Avoidance for Airport Traffic Concept Evaluation, Proceedings of the 28th Digital Avionics Systems Conference, Orlando, FL.

[8] Jones, D. R., L. J. Prinzel, K. J. Shelton, R. E. Bailey, S. D. Otero, and G. D. Barker, 2010, Collision Avoidance for Airport Traffic Simulation Evaluation, Proceedings of the 29th Digital Avionics Systems Conference, Salt Lake City, UT.

[9] Jones, D. R., R. C. Chartrand, S. R. Wilson, S. A. Commo, S. D. Otero, and G. D. Barker, 2012, Airport Traffic Conflict Detection and Resolution Algorithm Evaluation, Proceedings of the 31st Digital Avionics Systems Conference, Williamsburg, VA.

[10] Jones, D. R., R. C. Chartrand, S. R. Wilson, S. A. Commo, S. D. Otero, and G. D. Barker, 2012, SURF IA Conflict Detection and Resolution Algorithm Evaluation, Proceedings of the 31st Digital Avionics Systems Conference, Williamsburg, VA.

[11] Federal Aviation Administration, 2007, Electronic Flight Deck Displays, Advisory Circular 25-11A, Washington, DC.
[12] RTCA, Inc., 2001, User Requirements for Aerodrome Mapping Information, RTCA DO-272, Washington, DC.

[13] RTCA, Inc., 2010, Safety, Performance and Interoperability Requirements Document for Enhanced Traffic Situational Awareness on the Airport Surface with Indications and Alerts (SURF IA), DO-323, Washington, DC.

[14] Otero, S. D., G. D. Barker, and D. R. Jones, 2013, Initial Concept for Terminal Area Conflict Detection, Alerting, and Resolution Capability On or Near the Airport Surface, Version 2.0, NASA TM2013-218052, NASA Langley Research Center, Hampton, VA.

[15] Mohleji, S. C., and G. Wang, 2010, Modeling ADS-B Position and Velocity Errors for Airborne Merging and Spacing in Interval Management Application, MITRE release \# 10-3026.

[16] RTCA, Inc., 2002, Minimum Aviation System Performance Standards for Automatic Dependent Surveillance Broadcast (ADS-B), DO-242A, Washington, DC.

[17] Federal Aviation Administration, 2010, Automatic Dependent Surveillance - Broadcast (ADS-B) Out Performance Requirements to Support Air Traffic Control (ATC) Service, Final Rule, Docket No. FAA-2007-29305, Amdt. No. 91-314, Department of Transportation, Washington DC.

[18] RTCA, Inc., 2003, Minimum Aviation System Performance Standards for Aircraft Surveillance Applications (ASA), DO-289, Washington, DC.

[19] RTCA, Inc., 2010, Safety, Performance and Interoperability Requirements Document for ATSASURF Application, DO-322, Washington, DC.

\section{Acknowledgements}

The authors would like to express their appreciation to NASA LaRC Simulation Development and Analysis Branch personnel for their support throughout all phases of implementation and testing and to NASA's Aviation Safety Program for sponsoring this research.

33rd Digital Avionics Systems Conference October 5-9, 2014 